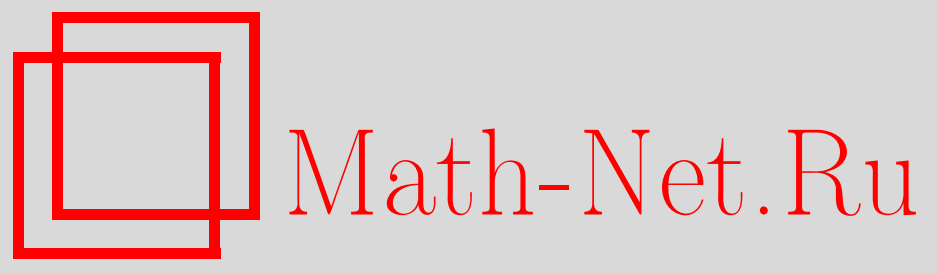

Н. Н. Попов, Л. В. Коваленко, Оценка надёжности осесимметричных стохастических элементов конструкций при ползучести по теории выбросов, Вестн. Сам. гос. техн. ун-та. Сер. Физ.-мат. науки, 2012, выпуск 2(), 72-77

DOI: https://doi.org/10.14498/vsgtu1090

Использование Общероссийского математического портала Math-Net.Ru подразумевает, что вы прочитали и согласны с пользовательским соглашением

http://www . mathnet.ru/rus/agreement

Параметры загрузки:

IP : 54.198 .67 .100

26 апреля 2023 г., 10:38:10

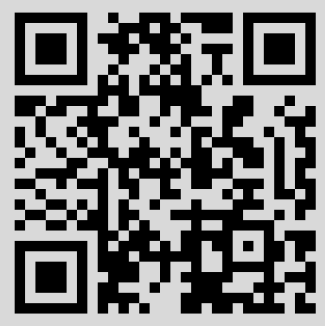


Вестн. Сам. гос. техн. ун-та. Сер. Физ.-мат. науки. 2012. № 2 (27). С. $72-77$

УДК 519.2:539.376

\title{
ОЦЕНКА НАДЁЖНОСТИ ОСЕСИММЕТРИЧНЫХ СТОХАСТИЧЕСКИХ ЭЛЕМЕНТОВ КОНСТРУКЦИЙ ПРИ ПОЛЗУЧЕСТИ ПО ТЕОРИИ ВЫБРОСОВ
}

\author{
Н. Н. Попов, Л.В. Коваленко \\ Самарский государственный технический университет, \\ 443100, Россия, Самара, ул. Молодогвардейская, 244. \\ E-mails: ponick25@gmail.ru; flytitmouse@mail.ru
}

\begin{abstract}
Рассмотрены вероятностные методы оценки прочностной надёжности осесимметричных элементов конструкиий, работающих в условиях ползучести. Реологические свойства материала при этом описывались при помощи случайной функиии одной переменной (радиуса $r$ ). Оценка надёжности осесимметричных стохастически неоднородных элементов конструкиий производилась по критерию деформационного типа. Ограничение накладывалось на случайное перемещение $w(r, t)$, зависящее от времени и пространственной координать. Считалосъ, что случайное неоднородное поле $w(r, t)$ имеет гауссовское распределение, для которого были сделаны некоторые упрощения. Пределъно допустимое значение перемещения считалось детерминированным. В качестве примера рассматривались модельные задачи по вычислению основной характеристики надёэности вероятности безотказной работы для микронеоднородной толстостенной трубъ с заданными параметрами, находящейся под действием внутреннего давления. Далее производилось сравнение вероятности безотказной работы и ресурса трубы, полученных в данной работе по критерию деформационного типа, и результатам, полученным другими авторами. Показано, что данный подход можно использовать для высоконадёжных конструкиий, когда выброс за критический уровень является достаточно редким собътием.
\end{abstract}

Ключевые слова: ползучесть, толстостенная труба, случайное перемещение, дебормационный критерий, теория выбросов.

Исследование напряжённо-деформированного состояния распределённых элементов конструкций путем решения стохастической краевой задачи является первым этапом расчёта элемента конструкции на надёжность. На втором этапе выявляются опасные зоны в элементах конструкций и на основе какоголибо критерия оценивается их работоспособность.

В данной работе рассматриваются вероятностные методы оценки прочностной надёжности осесимметричных элементов конструкций при ползучести, реологические свойства которых описываются при помощи случайной функции одной переменной (радиуса $r$ ).

Деформационный критерий отказа для таких элементов конструкций может быть сформулирован в виде некоторого соотношения для перемещения $w(r, t)$. Предельное значение перемещения $w_{0}$ предполагается детерминированной величиной. Если во всех точках элемента конструкции выполняется соотношение

$$
w(r, t)<w_{0}
$$

то условие прочности считается выполненным, элемент конструкции являет-

Николай Николаевич Попов (к.ф.-м.н., доц.), доцент, каф. прикладной математики и информатики. Людмила Викторовна Коваленко (к.ф.-м.н.), ассистент, каф. прикладной математики и информатики. 
ся работоспособным. При выполнении условия $w(r, t) \geqslant w_{0}$ хотя бы в одной точке происходит локальный отказ, что приводит к отказу всего элемента конструкции.

Основной количественной характеристикой надёжности является вероятность безотказной работы. Она в данном случае определяет вероятность того, что во всех точках элемента конструкции выполняется условие прочности (1), и математически записывается следующим образом [1]:

$$
P(t)=P\left\{\sup _{a \leqslant r \leqslant b} w(r, t)<w_{0}, \tau \in[0, t]\right\} .
$$

Поскольку перемещение любого фиксированного радиуса в условиях установившейся ползучести является возрастающей функцией по $t$, для вероятности безотказной работы $P(t)$ на отрезке времени $[0, t]$ имеет место более простая формула [1]:

$$
P(t)=P\left\{\sup _{a \leqslant r \leqslant b} w(r, t)<w_{0}\right\} .
$$

Вероятность безотказной работы (2) определяется лишь приближённо с использованием среднего числа выбросов функции $w(r, t)$ (при фиксированном $t$ ) за детерминированный уровень $w_{0}$. Если выброс случайной функции $w(r, t)$ за допустимый уровень $w_{0}$ является достаточно редким событием, то распределение выбросов можно считать пуассоновским. Тогда для вероятности безотказной работы (2) справедлива формула [1]

$$
P(t) \approx \exp \left(-\int_{1}^{\beta} \lambda(r, t) d r\right)
$$

где $\lambda(r, t)$ - математическое ожидание числа выбросов за уровень $w_{0}$ на единицу длины.

Вычисление величины $\lambda(r, t)$ существенно зависит от вида случайного поля $w(r, t)$. Значительное упрощение достигается для гауссовских (нормальных) однородных случайных полей [1]:

$$
\lambda(r, t)=\frac{\sqrt{D^{\prime}}}{2 \pi \sqrt{D}} \exp \left(-\frac{\left(w_{0}-a\right)^{2}}{2 D}\right),
$$

где $a=\langle w(r, t)\rangle, D$ - дисперсия случайной функции $w(r, t), D^{\prime}$ - дисперсия производной $\partial w / \partial r$.

Для гауссовского одномерного неоднородного случайного поля формула для вычисления среднего числа выбросов выглядит значительно сложнее [1]:

$$
\begin{aligned}
\lambda(r, t)=\frac{\sqrt{D^{\prime}}}{2 \pi} \sqrt{D} & \exp \left(-\frac{\left(w_{0}-a\right)^{2}}{2 D}\right) \times \\
\times & {\left[\sqrt{1-\rho^{2}} \exp \left(-\frac{1}{2\left(1-\rho^{2}\right)}\left(\frac{\left\langle v^{\prime}\right\rangle}{\sqrt{D^{\prime}}}-\frac{\rho\langle v\rangle}{\sqrt{D^{\prime}}}\right)^{2}\right)+\right.} \\
+ & \left.2 \pi\left(\frac{\left\langle v^{\prime}\right\rangle}{\sqrt{D^{\prime}}}-\frac{\rho\langle v\rangle}{\sqrt{D}}\right)\left(1-\Phi\left(-\frac{1}{\sqrt{1-\rho^{2}}}\left(\frac{\left\langle v^{\prime}\right\rangle}{\sqrt{D^{\prime}}}-\frac{\rho\langle v\rangle}{\sqrt{D}}\right)\right)\right)\right],
\end{aligned}
$$


где $\rho(r, t)$ - коэффициент корреляции между случайной функцией и её производной в одной и той же точке (при фиксированном времени):

$$
\rho(r, t)=\frac{1}{\sqrt{D} \sqrt{D^{\prime}}}\left(\left\langle w(r, t) w^{\prime}(r, t)\right\rangle-\langle w(r, t)\rangle\left\langle w^{\prime}(r, t)\right\rangle\right),
$$

$\Phi(x)$ - функция Лапласа, $\langle v(r, t)\rangle=\langle w(r, t)\rangle-w_{0},\left\langle v^{\prime}(r, t)\right\rangle=\left\langle w^{\prime}(r, t)\right\rangle$ (производная вычисляется по переменной $r)$.

Вычисление вероятности безотказной работы с помощью теории выбросов является наиболее адекватным реальным процессам. Однако в силу сложности вычисления вероятности по формулам (3), (4) для неоднородных случайных полей можно пойти к дальнейшему упрощению. Пусть $\sup _{a \leqslant r \leqslant b} w(r, t)$ достигается в точке максимума среднего значения $\langle w(r, t)\rangle$ (при фиксированном времени). Тогда формулу для вычисления вероятности безотказной работы (3) можно записать в виде

$$
P(t)=P\left\{w\left(r_{0}, t\right)<w_{0}\right\}=\int_{0}^{w_{0}} f(x, t) d x,
$$

где $r_{0}$ - точка, в которой функция $\langle w(r, t)\rangle$ принимает наибольшее значение; $f(x, t)$ - плотность распределения случайной величины $w\left(r_{0}, t\right)$.

Очевидно, формула (6) даёт приемлемую оценку вероятности безотказной работы в тех случаях, когда имеется единственная точка с ярко выраженным максимумом среднего значения $\langle w(r, t)\rangle$, а моменты второго и более высоких порядков малы.

В качестве примера рассмотрим оценку надёжности толстостенной трубы из микронеоднородного материала, находящейся под действием внутреннего давления $q$. Решение стохастической краевой задачи установившейся ползучести толстостенной трубы рассматривалось в работах [2,3]. Определяющие соотношения для деформаций ползучести $\varepsilon_{r}$ и $\varepsilon_{\varphi}$ принимались в соответствии с теорией вязкого течения в стохастической форме:

$$
2 \dot{\varepsilon}_{\varphi}=-2 \dot{\varepsilon}_{r}=c s^{n-1}\left(\sigma_{\varphi}-\sigma_{r}\right)(1+\alpha U(r)),
$$

где $\sigma_{r}$ и $\sigma_{\varphi}$ - радиальная и тангенциальная компоненты тензора напряжений; $U(r)$ - случайная функция, описывающая флуктуации реологических свойств материала; $\alpha \in(0,1)$ - коэффициент вариации этих свойств; $c, n-$ константы материала.

В работе [3] на основе второго приближения метода малого параметра найдено случайное перемещение

$$
w(r, t)=T \beta^{2} \frac{t a}{r}\left[1+\frac{2 \alpha}{n} H_{1}+\alpha^{2}\left(\frac{2(n+1)}{n^{3}} H_{1}^{2}-\frac{n+1}{n^{2}} H_{2}\right)\right],
$$

его математическое ожидание

$$
\langle w(r, t)\rangle=T \beta^{2} \frac{t a}{r}\left[1+\frac{2 \alpha}{n}\left\langle H_{1}\right\rangle+\alpha^{2}\left(\frac{2(n+1)}{n^{3}}\left\langle H_{1}^{2}\right\rangle-\frac{n+1}{n^{2}}\left\langle H_{2}\right\rangle\right)\right]
$$

и дисперсия 


$$
\begin{aligned}
D[w(r, t)]=T^{2} \beta^{4}\left(\frac{\operatorname{ta} \alpha}{r n}\right)^{2}[ & 4 D\left[H_{1}\right]+\frac{4(n+1)^{2} \alpha^{2}}{n^{4}} D\left[H_{1}^{2}\right]+ \\
& \left.+\frac{(n+1)^{2} \alpha^{2}}{n^{2}} D\left[H_{2}\right]-\frac{4(n+1)^{2} \alpha^{2}}{n^{3}}\left\langle\stackrel{\circ}{H}_{1}^{2} \stackrel{\circ}{H}_{2}^{2}\right\rangle\right] .
\end{aligned}
$$

Здесь $r$ - безразмерный радиус, $a$ и $b$ - внутренний и внешний радиусы трубы, $\beta=b / a, T=(\sqrt{3})^{(n-1)} c q^{n}\left(\beta^{2 / n}-1\right)^{-n} n^{-n}, q$ - давление, $\left\langle H_{2}\right\rangle=n / 2$,

$$
\left\langle H_{1}^{2}\right\rangle=\frac{1}{\left(1-\beta^{-2 / n}\right)^{2}} \int_{1}^{\beta} \int_{1}^{\beta} K\left(x_{2}-x_{1}\right)\left(x_{1} x_{2}\right)^{-1-2 / n} d x_{1} d x_{2}
$$

$K\left(x_{2}-x_{1}\right)$ - корреляционная функция случайной функции $U(r)$. Выражения для дисперсий $D\left[H_{1}\right], D\left[H_{1}^{2}\right], D\left[H_{2}\right]$ и центрального момента $\left\langle\stackrel{\circ}{H}_{1}^{2} \stackrel{\circ}{H}_{2}^{2}\right\rangle$ в силу их громоздкости здесь не выписаны.

Следует заметить, что в формулах (7)-(9) выражения в квадратных скобках не зависят ни от времени $t$, ни от безразмерной координаты $r$.

Модельная задача оценки надёжности для толстостенной трубы из стали $12 \mathrm{XM}\left(T=590^{\circ} \mathrm{C}\right)$ с постоянными материала $n=7,1, c=2,762 \cdot 10^{-21}$, внутренним и наружным радиусами $a=14$ мм и $b=21$ мм соответственно, находящейся под действием внутреннего давления $q=70 \mathrm{MПа,} \mathrm{рассматривалась}$ в работе [3]. Исходные данные взяты из монографии [4]. В качестве параметра, определяющего ресурс трубы, использовалось перемещение, критическое значение которого принималось равным $w_{0}=2$ мм. Вычисление надёжности производилось по упрощенной формуле (6), при этом предполагалось, что функция $w(r, t)$ подчинена нормальному закону, и разрушение начинается на внутреннем радиусе $(r=1)$, где $\langle w(r, t)\rangle$ принимает наибольшее значение. Для нормального закона формула (6) имеет вид

$$
P(t)=\frac{1}{\sqrt{2 \pi D[w(t)]}} \int_{0}^{w_{0}} \exp \left(-\frac{(x-\langle w(t)\rangle)^{2}}{2 D[w(t)]}\right) d x
$$

При степени неоднородности $\alpha=0,3$ в [3] было получено, что $\langle w(t)\rangle=9,191 \times$ $\times 10^{-5} t, D[w(t)]=1,768 \cdot 10^{-11} t^{2}$.

Рассмотрим теперь оценку надёжности трубы по формулам (3), (4). В силу того, что $\partial w(r, t) / \partial r=-w(r, t) / r^{2}$, коэффициент корреляции $\rho(r, t)$, определённый по формуле $(5)$, равен $(-1)$, и формула (4) значительно упростится:

$$
\lambda(r, t)=\frac{\sqrt{D^{\prime}}}{2 \sqrt{D}}\left(\frac{\left\langle v^{\prime}\right\rangle}{\sqrt{D^{\prime}}}-\frac{\rho\langle v\rangle}{\sqrt{D}}\right) \exp \left(-\frac{\left(w_{0}-a\right)^{2}}{2 D}\right) .
$$

На рисунке представлены функции надёжности $P(t)$ для трубы из стали 12ХМФ $\left(T=590^{\circ} \mathrm{C}\right)$, вычисляемые по формулам теории выбросов $(3)$, (11) и по упрощённой формуле (10): a) $a=14$ мм, $b=21 \mathrm{мм}, q=70$ МПа, $\alpha=0,3$ [3]; б) $a=14 \mathrm{mм}, b=16,68 \mathrm{Mм}, q=28 \mathrm{MПа,} \alpha=0,1$ [4]. Из полученных данных видно, что для высоконадёжных конструкций, когда выброс за критический уровень является достаточно редким событием (именно только для таких конструкций можно использовать формулу (3)), графики функций надёжности отличаются незначительно. 

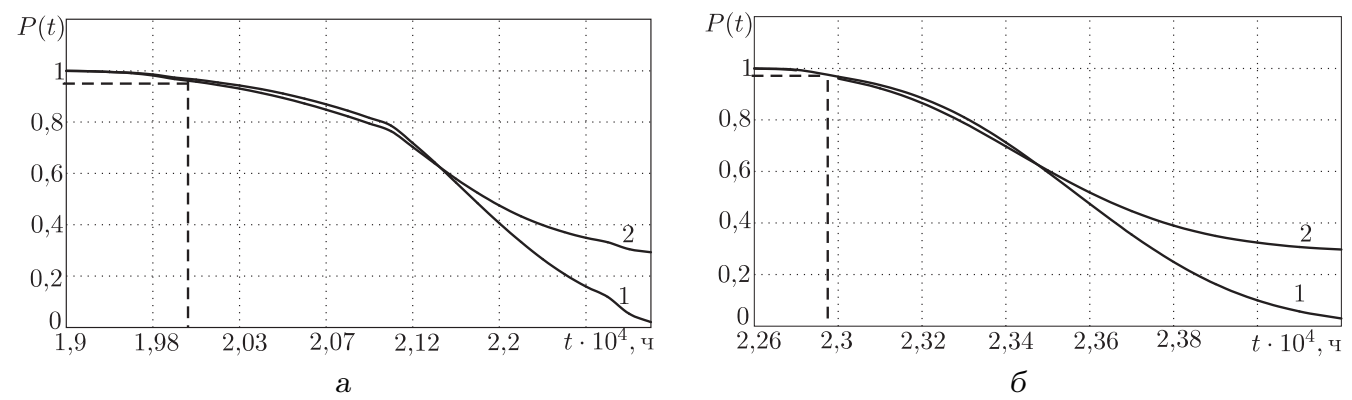

Функция надёжности $P(t)$ для толстостенной трубы: 1) вычисленная по упрощённой формуле $(10) ; 2)$ вычисленная по теории выбросов (формулы (3), (11))

Функцию надёжности $P(t)$ можно использовать для назначения ресурса толстостенной трубы. Назначенный ресурс $T_{*}$ определяют так, чтобы вероятность обеспечения $T_{*}$ была равна заданному значению $P_{*}$ вероятности безотказной работы (пунктирные линии на графиках). В первом примере (рис. а) при заданном значении $w_{0}=2$ мм ресурс для рассматриваемой трубы составляет 19972 ч с вероятностью $p_{*}=0,975$, вычисленной по формуле $(3)$, и с $p_{*}=0,969$, вычисленной по формуле (10). Для второго примера (рис. б) при заданном значении $w_{0}=1 \mathrm{мм} \mathrm{ресурс} \mathrm{составляет} 22964$ ч соответственно с вероятностями $p_{*}=0,975$ (формула $\left.(3)\right)$ и $p_{*}=0,968$ (формула $(10)$ ).

\section{БИБЛИОГРАФИЧЕСКИЙ СПИСОК}

1. Болотин B. В. Методы теории вероятностей и теории надёжности в расчетах сооружений. М.: Стройиздат, 1982. 352 с. [Bolotin V. V. Methods of the Probability Theory and Reliability Theory in Structural Design. Moscow: Stroyizdat, 1982. 352 pp.]

2. Должковой А.А., Попов Н.Н., Радченко В. П. Решение стохастической краевой задачи установившейся ползучести для толстостенной трубы методом малого параметpa// ПMTФ, 2006. Т.47, №1. С. 161-171; англ. пер.: Dolzhkovoi A. A., Popov N. N., Radchenko V.P. Solution of the Stochastic Boundary-Value Problem of Steady-State Creep for a Thick-Walled Tube Using the Small-Parameter Method // J. Appl. Mech. Tech. Phys., 2006. Vol. 47, no. 1. Pp. 134-142.

3. Исуткина В.Н. Разработка аналитических методов решения стохастических краевых задач установившейся ползучести для плоского деформированного состояния: Дисс. ... канд. физ.-мат. наук. Самара, 2007. 247 с. [Isutkina V.N. Development of analytical methods for solving stochastic boundary value problems of steady-state creep for a flat strain state: Ph. D. Thesis (Phys. \& Math.). Samara, 2007. 247 pp.]

4. Радченко В.П., Еремин Ю.А. Реологическое деформирование и разрушение материалов и элементов конструкций. М.: Машиностроение-1, 2004. 265 с. [Radchenko V.P., Eremin Yu. A. Rheological deformation and fracture of materials and structural elements. Moscow: Mashinostroenie-1, 2004. 265 pp.]

Поступила в редакцию 25/II/2012;

в окончательном варианте - 25/V/2012. 
MSC: 74S60; 74E05, 74E35, 74C10, 74R20

\title{
EVALUATION OF RELIABILITY OF AXISYMMETRIC STOCHASTIC ELEMENTS OF CONSTRUCTIONS UNDER CREEPAGE ON THE BASIS OF THEORY OF RUNS
}

\author{
N. N. Popov, L. V. Kovalenko
}

Samara State Technical University, 244, Molodogvardeyskaya st., Samara, 443100, Russia.

E-mails: ponick25@gmail.ru; flytitmouse@mail.ru

\begin{abstract}
Probabilistic methods of evaluating of strength reliability of construction axisymmetric elements of working in creep conditions were considered. Rheological properties of the material were described by the random function of one variable (of radius $r$ ). Evaluation of reliability of stochastically inhomogeneous axisymmetric structural elements was considered by deformation criterion. The restriction was imposed on random displacement, which depends on the time and spatial coordinates. It was assumed that the random field of function $w(r, t)$ has Gaussian distribution, for which some simplifications were made. Maximum valid value of displacement was deterministic (value was taken from the model problems). The calculation of probability of failure-free operation of microinhomogeneous thick-walled cylinder with given parameters under internal pressure $q$ was considered as an example. The probability of failure-free operation and service life of thick-walled cylinder obtained in this work by the deformation criterion and the results obtained by other authors was compared. It was shown that this approach can be used for fail-safe constructions, which critical level of runs is a rare event.
\end{abstract}

Key words: creep, thick-walled tube, random displacement, deformation criteria, theory of runs.

Original article submitted 25/II/2012; revision submitted $25 / \mathrm{V} / 2012$.

Nikolay N. Popov (Ph. D. (Phys. \& Math.)), Associate Professor, Dept. of Applied Mathematics \& Computer Science. Ludmila V. Kovalenko (Ph. D. (Phys. \& Math.)), Assistent, Dept. of Applied Mathematics \& Computer Science. 